\title{
BEDIENTHEORETISCHE MODELLIERUNG EINER BLECHTEILFERTIGUNG ALS MITTEL DES MATERIALFLUSSCONTROLLINGS
}

\author{
Kai Furmans, Universität Karlsruhe \\ Rolf Soukal, RWTH Aachen
}

Die Planung und Steuerung eines Fertigungsbereiches, in dem viele verschiedene Produkte in mehreren schritten auf eine große Zahl von verschiedenen Maschinen hergestellt werden, ist eine komplexe Aufgabe. Insbesondere der zusammenhang zwischen vorhandener Kapazität, erwartetem Durchsatz und der hierfür notwendigen Durchlaufzeit, die gleichzeitig auch den Umlaufbestand bestimmt, ist häufig Gegenstand von Diskussionen. Deshalb wird vorgeschlagen, bedientheoretische Methoden einzusetzen, um ein akzeptables Niveau für Umlaufbestand und Durchlaufzeit unter Berücksichtigung der aktuellen Kapazitätsauslastung zu bestimmen.

Um die Verwendbarkeit dieses Ansatzes zu überprüfen, wurde eine Blechteilfertigung bei einem Automobilhersteller zwei Monate beobachtet. Während dieser zeit durchliefen etwa 800 Aufträge die 35 Maschinen und Maschinengruppen in diesem Fertigungsabschnitt. Die Bearbeitungs- und die Wartezeiten an jeder Maschine wurden für jeden Auftrag aufgezeichnet. Dazu kamen Brach- und Störzeiten der Maschinen. Auf der Basis dies:=r Informationen wurde ein geschlossenes und ein offenes Bediensystemnetzwerk modelliert.

Bei der Modellierung mußte einigen Besonderheiten Rechnung getragen werden. Die Maschinen wurden in verschiedenen Schichtmodellen betrieben. Dies führt zu zusätzlichen Pufferzeiten, die sich zu den Wartezeiten addieren, die aus den zugrundeliegenden stochastischen Prozessen resultieren. Gleichzeitig wird dadurch die korrekte Berechnung des Auslastungsgrades erschwert. Ein weiteres Problem stellt die gelegentliche Aufteilung von großen Aufträgen dar, die dann parallel auf mehreren Maschinen bearbeitet werden.

Die Kennwerte für die bedientheoretischen Modelle wurden mit Hilfe des Programmpaketes PEPSY berechnet, das von $G$. Bolch und seiner Gruppe von der Universität Erlangen-Nürnberg zur Verfügung gestellt wurde. Hiermit konnten verschiedene Näherungsverfahren verwendet und verglichen werden. Die Analyse der Ergebnisse zeigt, daß Kennwerte für das Gesamtsystem mit hinreichendér Genauigkeit bestimmt werden können. Bei Werten, die sich auf einzelne Maschinen beziehen, mußten zum Teil große Abweichungen festgestellt werden. Diese konnten zum Teil auf abweichende Abarbeitungsreihenfolgen zurückgeführt werden. Es zeigte sich jedoch auch, daß es notwendig ist, die Approximationsverfahren weiter zu verbessern, um auch beispielsweise mehrgipflige Bedienzeitverteilungen besser abzubilden. 